\title{
Game Semantics for Constructive Modal Logic
}

\author{
Matteo Acclavio ${ }^{1}$, Davide Catta ${ }^{2}$, and Lutz Straßburger ${ }^{3}$ \\ 1 University of Luxembourg, Belval, Luxembourg \\ 2 LIRMM - Université de Montpellier, Montpellier, France \\ 3 INRIA-Saclay and LIX-École polytechnique, Palaiseau, France
}

\begin{abstract}
In this paper we provide the first game semantics for the constructive modal logic CK. We first define arenas encoding modal formulas, we then define winning innocent strategies for games on these arenas, and finally we characterize the winning strategies corresponding to proofs in the logic CK. To prove the full-completeness of our semantics, we provide a sequentialization procedure of winning strategies. We conclude the paper by proving their compositionality and showing how our results can be extend to the constructive modal logic CD.
\end{abstract}

\section{Introduction}

Modal logics are extensions of classical logic making use of modalities to qualify the truth of a judgement. According to the interpretation of such modalities, modal logics find applications, for example, in knowledge representation [28], artificial intelligence [19] and formal verification [10]. More precisely, modal logics are obtained by extending classical logic with a modality operator $\square$ (together with its dual operator $\diamond$ ), which are usually interpreted as necessity (respectively possibility).

When we move from the classical to the intuitionistic setting, the modality $\diamond$ is no longer the dual of the modality $\square$ and by consequence the the classical k-axiom $\square(A \supset B) \supset(\square A \supset \square B)$ is no longer sufficient to express the behavior of the modality $\diamond$. Depending on the chosen axioms, it is possible to define different flavors of "intuitionistic modal logics" (see, e.g., [8/23|22|24|4[6]). In this paper we consider the minimal approach obtained by adding only the axiom $\square(A \supset B) \supset(\diamond A \supset \diamond B)$, leading to what in the literature is now called constructive modal logic CK [23]4|9|18]7]13].

The study of the semantics of proofs in this logic is still rough and the only full complete denotational model for this logic is defined by the quotient of its $\lambda$-calculus with respect to $\beta$-reduction [3/4]. The purpose of this paper is to provide a full complete denotational semantics for CK in terms of a game semantics [1111|17]. Thereby we provide a concrete denotational model for this logic, that is, a model whose elements are not obtained by the quotient on proofs induced by cut-elimination.

In game semantics proofs are denoted by winning strategies for two-player games played on a graph, called modal arena, that encodes a modal formula. We denote the players by $\circ$ (white) and $\bullet$ (black). In the literature the white player is called opponent (denoted by $\mathrm{O}$ ) and the black player is called proponent (denoted by $\mathrm{P}$ ). The motivations of our choices is due to the correspondence between players' moves, the parity of the depth in the corresponding vertex in the modal arena ( $\circ$ for even and $\bullet$ for odd), and the 


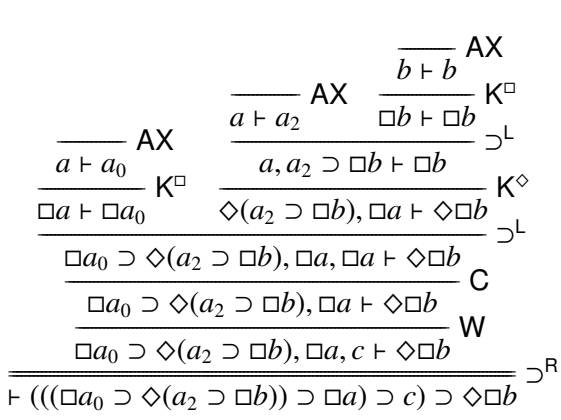
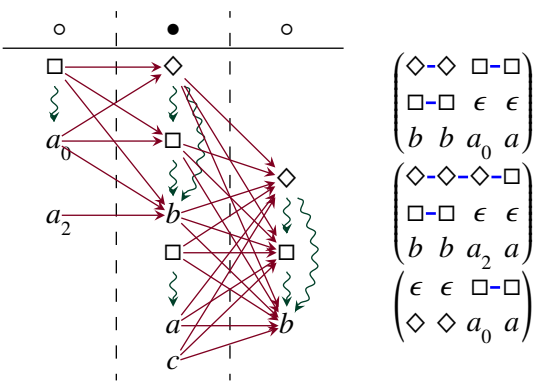

Fig. 1. A derivation $\mathcal{D}$ of the formula $F=\left(\left(\left(\square a_{0} \supset \diamond\left(a_{2} \supset \square b\right)\right) \supset \square a\right) \supset c\right) \supset \diamond \square b$, the modal arena $\llbracket F \rrbracket$, and the maximal batched views in the CK-WIS $\{\{\mathcal{D}\}\}$ of $F$. We indexed some occurrences of the atom $a$ to avoid ambiguity in the views.

polarities of the corresponding atoms in a polarized sequent calculus [15]16]2] where $\circ$ and $\bullet$ are usually used respectively for the positive and negative polarities.

Each play consists of an alternation of o-moves and $\bullet$-moves, that is, a play is represented by a list of occurrences of the vertices in the modal arena. The first move in a play is a o-move selected among the $\rightarrow$-roots of the modal arena. Each subsequent move of a player must be justified by a previous move of the other player, that is, the selected vertex must be the source of a $\rightarrow$-edge with target a vertex previously played by the other player. The game terminates when one player has no possible moves, losing the play. A winning innocent strategy (for $\bullet$ ) is a set of plays which takes into account every possible o-move, while each •-move is uniquely determined (and justified) by one of the previous o-moves. Intuitively, a winning strategy is a complete description of all the the possible plays always leading to the victory of $\bullet$. The adjective innocent is referred to the play-style of $\circ$ which chooses each of its non-initial moves only according with the previous $\bullet$-move in the play.

In [26] it is shown how the syntax of intuitionistic combinatorial proofs, a graphical proof system for propositional intuitionistic logic, provides intuitive insights about the winning innocent strategies (or WISs) in a Hyland-Ong arena [11/21]. Following this intuition, in [2] we developed the syntax of intuitionistic combinatorial proofs for constructive modal logics allowing us to characterize the winning innocent strategies for this logic by extending this correspondence (see Figure 1).

De facto, the presence of the modal axioms leads to the need of a new notion of batches ${ }^{4}$ in a play in order to characterize winning innocent strategies corresponding to proofs in the constructive modal logic CK. By means of example consider the formulas $\square a \supset a$ and $(\square a \supset \square b) \supset(\square(a \supset b))$ which are not provable in CK. Their corresponding modal arenas are pictured below together with the unique maximal view in their winning innocent strategies. Instead of representing these views, we represent the corresponding batched views, which are matrices containing the view together with a decoration of each move given by the modalities in whose scope they occur.

\footnotetext{
${ }^{4}$ Batches can be interpreted as the nesting of a nested sequent calculi [5|25].
} 


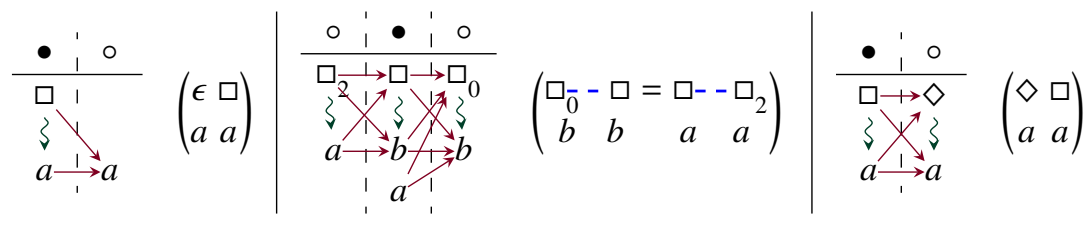

The strategies containing these views cannot be considered satisfactory since the modalities are not "properly batched" with respect to the modal rules in the sequent calculus for CK. In fact, the WIS containing these maximal views correspond to correct proofs in the intuitionistic propositional logic of the formulas obtained by removing the modalities, that is, $a \supset a$ and $(a \supset b) \supset(a \supset b)$.

In order to recover the correspondence between winning strategies and proofs, it suffices to consider two additional constraints on the accepted •-moves. We observe that each modality has a parity (the same of the corresponding node in the modal arena) and a height (defined as the number of the modalities in whose scope it belongs). The first constraint demands that each •-move must be in the scope of the same number of modalities of the previous o-move, ruling out the leftmost example in Equation (1). This constraint allows us to define sub-plays (corresponding to sub-proofs): whenever a o-move is in the scope of a new o-modality, that is, a modality whose scope contains no previous moves of the play, then the successive moves are played in a same sub-play. A sub-play ends when a o-move is in the scope of no modalities or in the scope of a new o-modality with equal or smaller height with respect to the previous $\bullet-$ move. Note that sub-plays can be nested. This allows us to gather modalities having the same height and in whose scope there are moves of a sub-play into batches. The second constraint demands that these batches have a specific shape, that is, the same of the modalities in the rules of the sequent calculus: only one $\circ$ modality occurs, and either all modalities are boxes or there is exactly one •-diamond and one o-diamond. These conditions rule out the existence of winning strategies for the formulas from Equation (1): in the first one the •-move has not the same height of the previous o-move, in the second one all the modalities are batched in the same set, which includes two o-modalities, in the third one the $\diamond^{\circ}$ does not have the corresponding $\diamond^{\bullet}$ in its batch.

Contribution of the paper. In this paper we show a direct correspondence between the sequent system for CK and our winning innocent strategies (CK-WIS). In particular, we show that the CK-WISs form a full-complete semantics for this logic. We then conclude the paper by showing that CK-WISs are a denotational semantics by proving their compositionality.

Organisation of the paper. In Section 2 we recall the definition of the constructive modal logic CK, its sound and complete sequent calculus and we recall the results from [2] on the encoding of modal arenas. In Section 3 we recall characterization of winning strategies encoding CK-proofs from [2] by providing a new detailed sequentialization procedure assuring the full-completeness of our model. In Section 4 we prove that our winning strategies compose. In Section 5 we collect the results allowing us to prove that we indeed define a full-complete denotational semantics for CK and we conclude in section Section 6 where we discuss related works and some future research directions. 


$$
\begin{array}{ccccc|c}
\frac{}{a \vdash a} \mathrm{AX} & \frac{\Gamma, B \vdash A}{\Gamma \vdash B \supset A} \supset^{\mathrm{R}} & \frac{\Gamma, B, C \vdash A}{\Gamma, B \wedge C \vdash A} \wedge^{\mathrm{L}} & \frac{\Gamma \vdash A \quad \Delta \vdash B}{\Gamma, \Delta \vdash A \wedge B} \wedge^{\mathrm{R}} & \frac{\Gamma \vdash A \quad \Delta, B \vdash C}{\Gamma, \Delta, A \supset B \vdash C} \supset^{\llcorner} & \frac{\Gamma \vdash A \quad \Delta, A \vdash B}{\Gamma, \Delta \vdash B} \text { cut } \\
\leftarrow_{1} 1 & \frac{\Gamma, B, B \vdash A}{\Gamma, B \vdash A} \mathrm{C} & \frac{\Gamma \vdash A}{\Gamma, B \vdash A} \mathrm{~W} & \frac{\Gamma \vdash A}{\square \Gamma \vdash \square A} \mathrm{~K}^{\square} & \frac{B, \Gamma \vdash A}{\diamond B, \square \Gamma \vdash \diamond A} \mathrm{~K}^{\diamond} &
\end{array}
$$

Fig. 2. The rules for the sequent system LCK and the cut-rule

\section{Background}

In this section we recall some basic definition for the constructive modal logic CK together with some extensions of the definition from [2] allowing to encode formulas by means of specific directed graphs we call modal arenas.

\subsection{Constructive modal logic}

We consider the (modal) formulas generated by a countable set of (atomic) propositional variables $\mathcal{A}=\{a, b, \ldots\}$ and the following grammar

$$
A, B::=a|1| A \supset B|A \wedge B| \square A \mid \diamond A
$$

We define the size $\|A\|$ of a formula $A$ as the number of connectives and modalities in $A$ and if $\Gamma=A_{1}, \ldots, A_{n}$ then $\|\Gamma\|=\sum_{i=1}^{n}\left\|A_{i}\right\|$. We say that a formula is modalityfree (respectively unit-free) if it contains no occurrences of $\square$ and $\diamond$ (respectively no occurrences of 1). A formula is a $\supset$-formula (resp. a $\wedge$-formula) if it is a formula of the form $A \supset B$ (resp. $A \wedge B$ ).

We define the formula isomorphism as the equivalence relation $\stackrel{f}{\sim}$ over formulas generated by the following relations:

$$
\begin{array}{cccc}
A \wedge 1 \stackrel{\mathrm{f}}{\sim} A & A \supset 1 \stackrel{\mathrm{f}}{\sim} 1 & 1 \supset A \stackrel{\mathrm{f}}{\sim} A & \square 1 \stackrel{\mathrm{f}}{\sim} 1 \\
A \wedge B \stackrel{\mathrm{f}}{\sim} B \wedge A \quad A \wedge(B \wedge C) \stackrel{\mathrm{f}}{\sim}(A \wedge B) \wedge C & (A \wedge B) \supset C \stackrel{\mathrm{f}}{\sim} A \supset(B \supset C)
\end{array}
$$

The constructive modal logic CK is obtained by extending the propositional intuitionistic logic [27] with the necessitation rule: "if $F$ is provable, then so is $\square F$ ", and the the following two modal axioms:

$$
\mathrm{k}_{1}: \square(A \supset B) \supset(\square A \supset \square B) \quad \mathrm{k}_{2}: \square(A \supset B) \supset(\diamond A \supset \diamond B)
$$

The sequent system LCK, given in Figure 2 , is a sound and complete proof system for the logic CK [14]. We write $\stackrel{\text { LCK }}{-} F$ whenever $\vdash F$ is provable in LCK.

Theorem 2.1. A formula $F$ is provable in $\mathrm{LCK} \cup\{\mathrm{cut}\}$ iff is provable in $\mathrm{LCK}$.

\subsection{Modal Arenas}

A directed graph $\mathcal{G}=\left\langle V_{\mathcal{G}}, \stackrel{\mathcal{G}}{\rightarrow}\right\rangle$ is given by a set of vertices $V_{\mathcal{G}}$ and a set of direct edges $\stackrel{\mathcal{G}}{\rightarrow} \subseteq V_{\mathcal{G}} \times V_{\mathcal{G}}$. A vertex $v$ is a $\stackrel{\mathcal{G}}{\rightarrow}$-root, denoted $v \nrightarrow$ if there is no vertex $w$ such that 
$v \stackrel{\mathcal{G}}{\rightarrow} w$. We denote by $\vec{R}_{\mathcal{G}}$ the set of $\stackrel{\mathcal{G}}{\rightarrow}$-roots of $\mathcal{G}$. A path from $v$ to $w$ of length $n$ is a sequence of vertices $x_{0} \ldots x_{n}$ such that $v=x_{0}, w=x_{n}$ and $x_{i} \stackrel{\mathcal{G}}{\rightarrow} x_{i+1}$ for $i \in\{0, \ldots, n-1\}$. We write $v \stackrel{\mathcal{G}}{\rightarrow}{ }^{n} w$ if there is a path from $v$ to $w$ of length $n$. A directed acyclic graph (or dag for short) is a direct graph such that $v \stackrel{\mathcal{G}}{\rightarrow}{ }^{n} v$ implies $n=0$ for all $v \in V$.

A two-color directed acyclic graph (or 2-dag for short) $\mathcal{G}=\left\langle V_{\mathcal{G}}, \stackrel{\mathcal{G}}{\rightarrow}, \stackrel{\mathcal{G}}{\rightsquigarrow}\right\rangle$ is given by a set of vertices $V_{\mathcal{G}}$ and two disjoint sets of edges $\stackrel{\mathcal{G}}{\rightarrow}$ and $\stackrel{\mathcal{G}}{\rightarrow}$ such that the graph $\left\langle V_{\mathcal{G}}, \stackrel{\mathcal{G}}{\rightarrow} \cup \stackrel{\mathcal{G}}{\rightsquigarrow}\right\rangle$ is acyclic. We omit the superscript when clear from context and we denote by $\emptyset$ the empty 2-dag.

If $\mathcal{L}$ is a set, a 2 -dag is $\mathcal{L}$-labeled if a label $\ell(v) \in \mathcal{L}$ is associated to each vertex $v \in V$. In this paper we fix the set of labels to be the set $\mathcal{L}=\mathcal{A} \cup\{\square, \diamond\}$, where $\mathcal{A}$ is the set of propositional variables occurring in formulas. We use the notation $a$, $\square$ and $\diamond$ to denote the graphs consisting of a single vertex labeled respectively by $a, \square$ and $\diamond$, and we denote by $V_{\mathcal{G}}^{\mathcal{A}}, V_{\mathcal{G}}^{\square}$ and $V_{\mathcal{G}}^{\diamond}$ the set of vertices of a graph $\mathcal{G}$ with labels respectively in $\mathcal{A},\{\square\}$ and $\{\diamond\}$.

Definition 2.2. Let $\mathcal{G}, \mathcal{H}$ and $\mathcal{F} \neq \emptyset$ be 2-dags, we denote by $R_{\mathcal{F}}^{\mathcal{G}}$ the set of edges from the $\rightarrow$-roots of $\mathcal{G}$ to the $\rightarrow$-roots of $\mathcal{F}$, that is $R_{\mathcal{F}}^{\mathcal{G}}=\left\{(u, v) \mid u \in \vec{R}_{\mathcal{G}}, v \in \vec{R}_{\mathcal{F}}\right\}$.

We define the following operations on 2-dags:

$$
\begin{aligned}
& \mathcal{G}+\mathcal{H}=\left\langle V_{\mathcal{G}} \cup V_{\mathcal{H}}, \stackrel{\mathcal{G}}{\rightarrow} \cup \stackrel{\mathcal{H}}{\rightarrow} \quad, \stackrel{\mathcal{G}}{\rightsquigarrow} \cup \stackrel{\mathcal{H}}{m} \quad\right\rangle \\
& \mathcal{G} \rightarrow \mathcal{F}=\left\langle V_{\mathcal{G}} \cup V_{\mathcal{F}}, \stackrel{\mathcal{G}}{\rightarrow} \cup \stackrel{\mathcal{F}}{\rightarrow} \cup R_{\mathcal{F}}^{\mathcal{G}}, \stackrel{\mathcal{G}}{m} \cup \stackrel{\mathcal{F}}{m} \quad\right\rangle \\
& \mathcal{G} \leadsto \mathcal{F}=\left\langle V_{\mathcal{G}} \cup V_{\mathcal{F}}, \stackrel{\mathcal{G}}{\rightarrow} \cup \stackrel{\mathcal{F}}{\rightarrow} \quad, \stackrel{\mathcal{G}}{\rightarrow} \cup \stackrel{\mathcal{F}}{m} \cup R_{\mathcal{F}}^{\mathcal{G}}\right\rangle \\
& \mathcal{G} \rightarrow \emptyset=\emptyset \quad \square \sim \emptyset=\emptyset \quad \diamond \sim \emptyset=\diamond
\end{aligned}
$$

which can be pictured as follows, with $\neg$ representing the $\rightarrow$-roots of each graph.

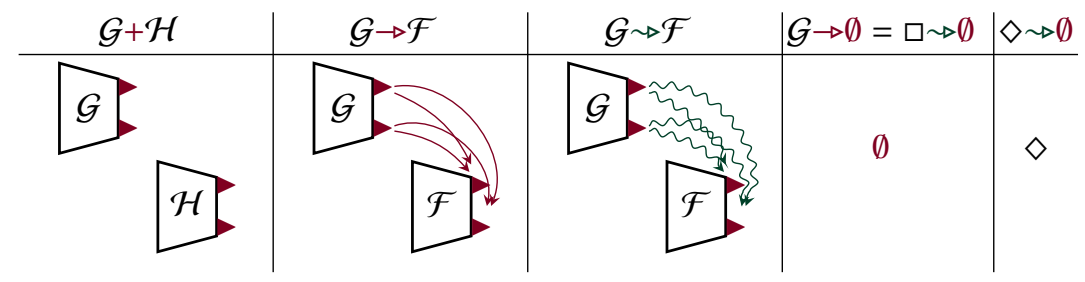

We can associate to each formula $F$ a $\mathcal{L}$-labeled 2-dag $\llbracket F \rrbracket$ as follows:

$$
\begin{aligned}
& \llbracket a \rrbracket=a \quad \llbracket A \supset B \rrbracket=\llbracket A \rrbracket \rightarrow \llbracket B \rrbracket \quad \llbracket A \wedge B \rrbracket=\llbracket A \rrbracket+\llbracket B \rrbracket \quad \llbracket 1 \rrbracket=\emptyset \\
& \llbracket \square A \rrbracket=\square \sim \llbracket A \rrbracket \quad \llbracket \diamond A \rrbracket=\diamond \sim \llbracket A \rrbracket
\end{aligned}
$$

Moreover, if $\Gamma \vdash A$ is a sequent, we denote by $\llbracket \Gamma \vdash A \rrbracket$ the modal arena $\llbracket\left(\bigwedge_{B \in \Gamma} B\right) \supset A \rrbracket$.

Definition 2.3. A modal arena is a $\mathcal{L}$-labeled 2-dag $\mathcal{G}$ such that $\mathcal{G}=\llbracket F \rrbracket$ for a modal formula $F_{5}^{5}$

\footnotetext{
${ }^{5}$ A geometrical characterization of the $\mathcal{L}$-labeled 2-dags which are modal arenas is out of the scope of this paper and can be found in [2].
} 
In this paper we may say that a vertex in $\llbracket F \rrbracket$ corresponds to an occurrence of atom or modality in a formula $F$, or we may identify them. An atomic vertex is a vertex corresponding to an atom, and a modal vertex in a vertex corresponding to a modality.

Definition 2.4. Let $\mathcal{G}=\llbracket F \rrbracket$ be a modal arena and $v \in V_{\mathcal{G}}$. The address of $v$ is the unique sequence of modal vertices $\operatorname{add}_{v}=m_{1}, \ldots, m_{h}$ in $V_{\mathcal{G}}$ which corresponds to the sequence of modalities in the path in the formula tree of $F$ connecting the node of $v$ to the root of $F$. If $\operatorname{add}_{v}=m_{1}, \ldots, m_{h}$, we denote by add ${ }_{v}^{k}=m_{k}$ its $k^{\text {th }}$ element and we call $h_{v}=\left|\operatorname{add}_{v}\right|$ the heigh of $v$, that is, the length of $\operatorname{add}_{v}$.

Example 2.5. Consider the modal arena and the formula tree of $(a \supset \square(b \wedge(c \supset$ $\left.\left.\left.\diamond_{1} d\right)\right)\right) \supset \diamond_{2}(e \supset f)$, then
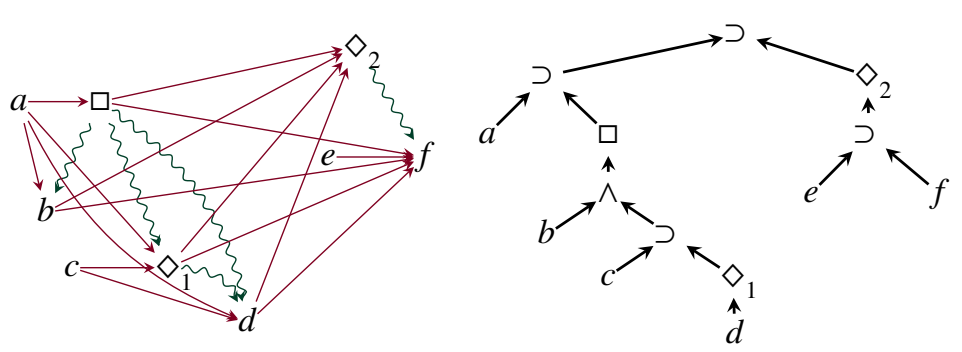

$$
\begin{aligned}
\operatorname{add}_{a} & =\epsilon \\
\operatorname{add}_{\square} & =\epsilon \\
\operatorname{add}_{b} & =\square \\
\operatorname{add}_{c} & =\square \\
\operatorname{add}_{\diamond_{1}} & =\square \\
\operatorname{add}_{d} & =\diamond_{1} \square \\
\operatorname{add}_{\diamond_{2}} & =\epsilon \\
\operatorname{add}_{e} & =\diamond_{2} \\
\operatorname{add}_{f} & =\diamond_{2}
\end{aligned}
$$

If $\mathcal{G}$ is a modal arena and $v \in V_{\mathcal{G}}$, we define $d(v)$ as the length of the $\rightarrow$-paths from $v$ to a $\rightarrow$-root $w \in \vec{R}_{\mathcal{G}}$. Note that the property that all paths in a modal arena from a vertex to any root have the same length is not trivial, but the proof can be found in [26, Lemma 9]. The parity of a vertex $v$ is the parity of $d(v)$, which can be either even or $o d d$. We denote by $v^{\circ}$ and $v^{\bullet}$ if the parity of $v$ is respectively even or odd. Note that the players $\circ$ and $\bullet$ can only play vertices of the corresponding parity, but the parity of the modalities in which the vertex belongs may not be the same as the parity of the move. By means of example, consider the atom $a_{2}$ in Figure 1 which is o but it is the scope of two $\bullet$-modalities.

We conclude the section by remarking that modal arenas identify formulas modulo the formula isomorphism $\stackrel{f}{\sim}$ defined by the relations in Equation 2 ,

Proposition 2.6. If $F$ and $G$ are two formulas, then $F \stackrel{f}{\sim} G \Longleftrightarrow \llbracket F \rrbracket=\llbracket G \rrbracket$.

Proof. If follows form the definition of the modal arenas operations,$+ \rightarrow$ and $\sim$.

\section{Winning Strategies for CK}

In this section we recall the definition of winning innocent strategy and we characterize the ones corresponding to correct CK-proofs. We then provide a direct proof of the correspondence between our winning innocent strategies and LCK-proofs by giving a desequentialization and a sequentialization procedure. The first procedure describes how to inductively define a winning strategy from a sequent calculus derivation. The second procedure defines a method to reconstruct a derivation in sequent calculus using the information contained in the winning strategy (and the proven formula). 
Definition 3.1. Let $F$ be a formula. A move is a vertex of $\llbracket F \rrbracket$. Let $\mathrm{p}=\mathrm{p}_{0} \cdots \mathrm{p}_{n}$ be a sequence of distinct moves (we denote by $\epsilon$ the empty sequence). If $v$ and $w$ are two moves in $\mathrm{p}$, we say that $w$ justifies $v$ whenever $v \stackrel{\llbracket F \|}{\rightarrow} w$. We call a move $\mathrm{p}_{i}$ in $\mathrm{p}$ a o-move or --move if $i$ is respectively even or odd.

We say that $p$ is a view in $\llbracket F \rrbracket$ if the following conditions are fulfilled:

1. $\mathrm{p}$ is a play: if $\mathrm{p} \neq \epsilon$, then $\mathrm{p}_{0} \in \vec{R}_{\|F\|}$;

2. $\mathrm{p}$ is justified: if $i>0$, then $\mathrm{p}_{i} \stackrel{\|F\|}{\rightarrow} \mathrm{p}_{i-(2 k+1)}$ for a $k \in \mathbb{N}$;

3. $\mathrm{p}$ is o-shortsighted: if $\mathrm{p}_{i+1}^{\circ}$ and $\mathrm{p}_{i}^{\circ}$, then $\mathrm{p}_{i+1} \stackrel{\|F\|}{\rightarrow} \mathrm{p}_{i}$;

4. $\mathrm{p}$ is $\bullet$-uniform: if $\mathrm{p}_{i+1}^{\bullet}$ and $\mathrm{p}_{i}^{\circ}$, then $\ell\left(\mathrm{p}_{i+1}\right)=\ell\left(\mathrm{p}_{i}\right)$;

5. $\mathrm{p}$ is modal: $\mathrm{p}_{i} \in V_{\llbracket F \rrbracket}^{\mathcal{A}} \cup V_{\llbracket F \|^{\diamond}}^{\diamond}$

Moreover, if $\mathrm{p}$ is a view, we say that

6. $\mathrm{p}$ is well-batched: $\left|\operatorname{add}_{p_{2 k}}\right|=\left|\operatorname{add}_{p_{2 k+1}}\right|$ for every $2 k \in\{0, \ldots, n-1\}$.

The predecessor of a non-empty view $\mathrm{p}$ is the sequence obtained by removing the last move in $\mathrm{p}$. The successor is the converse relation. A winning innocent strategy (or WIS for short) for $F$ (or over $\llbracket F \rrbracket$ ) is a finite non-empty set $\mathcal{S}$ of views in $\llbracket F \rrbracket$ such that:

a. $\mathcal{S}$ is predecessor-closed: if $p \cdot v \in \mathcal{S}$ then $p \in \mathcal{S}$;

b. $\mathcal{S}$ is o-complete: if $\mathrm{p} \in \mathcal{S}$ has even length, then every successor of $\mathrm{p}$ is in $\mathcal{S}$;

c. $\mathcal{S}$ is $\bullet$-deterministic and $\bullet$-total: if $\mathrm{p} \in \mathcal{S}$ has odd length, then exactly one successor of $\mathrm{p}$ is in $\mathcal{S}$.

A view is maximal in $\mathcal{S}$ if it is not prefix of any other view in $\mathcal{S}$. We say that a WIS $\mathcal{S}$ is trivial if $\mathcal{S}=\{\epsilon\}$ and it is well-batched if all its views are.

Note that our definition of WIS on arenas of modality-free formulas is the same of the one given in [11|21|26] where the modal condition trivially holds. Moreover, it follows by definition of view (by the fact that is a play, justified and o-shortsighted) that o-moves and $\bullet$-moves can only be vertices with the corresponding parity.

Remark 3.2. If $\mathcal{G}$ is a non-empty modal arena, then a WIS $\mathcal{S}$ on $\mathcal{G}$ must contain all views of the form $v$ with $v \in \vec{R}_{\mathcal{G}}$, that is, $\mathcal{S}$ is non-trivial.

Definition 3.3. Let $\mathrm{p}=\mathrm{p}_{0} \cdots \mathrm{p}_{n-1}$ be a view on a modal arena $\mathcal{G}$. We write $h_{\mathrm{p}}=$ $\max \left\{h_{v} \mid v \in \mathrm{p}\right\}$ and we define the batched view of $\mathrm{p}$ as the $h_{\mathrm{p}} \times n$ matrix $\mathcal{F}(\mathrm{p})=$ $\left(\mathcal{F}(\mathrm{p})_{0}, \ldots, \mathcal{F}(\mathrm{p})_{n}\right)$ with elements in $V_{\mathcal{G}} \cup\{\epsilon\}$ such that each column $\mathcal{F}(\mathrm{p})_{i}$ is defined as follows:

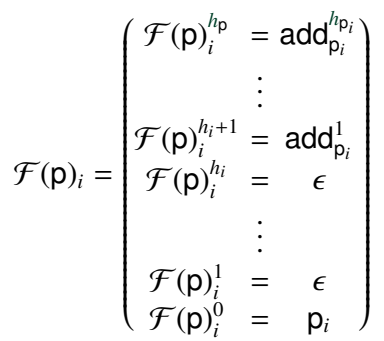




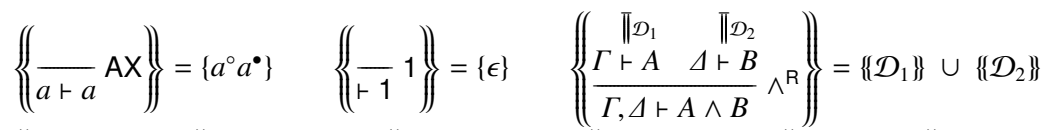

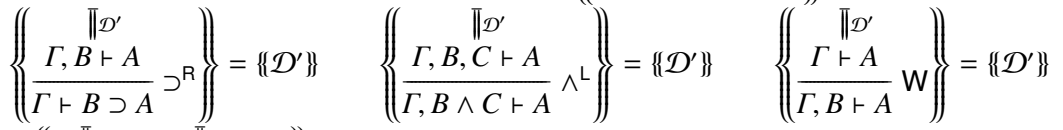

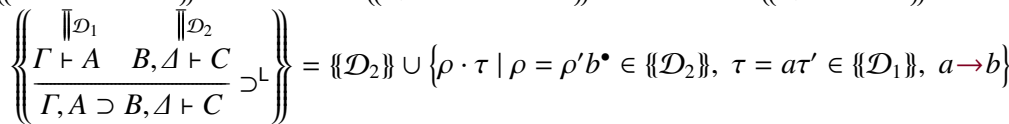

$$
\begin{aligned}
& \left.\left\{\left\{\frac{\Gamma, B, B \vdash A}{\Gamma, B \vdash A} \mathrm{U}\right\}\right\}=\left\{f(\sigma) \mid \sigma \in\left\{\mathcal{D}^{\prime}\right\}\right\} \text { and } f: V_{\llbracket \Gamma, B, B \vdash A \rrbracket} \rightarrow V_{\llbracket \Gamma, B \vdash A \rrbracket} \text { identifies the vertices on the } B \text { 's }\right\} \\
& \left\{\left(\begin{array}{c}
\| \mathcal{D}^{\prime} \\
\frac{\Gamma \vdash A}{\square \Gamma \vdash \square A} K^{\square}
\end{array}\right\}=\left\{\left\{\mathcal{D}^{\prime}\right\}\right\} \quad\left\{\begin{array}{c}
\| \mathcal{D}^{\prime} \\
\frac{\Gamma, B \vdash A}{\square \Gamma, \diamond^{\bullet} B \vdash \diamond^{\circ} A} K^{\square}
\end{array}\right\}=\left\{\left\{\mathcal{D}^{\prime}\right\} \cup\left\{\diamond^{\circ} \diamond^{\bullet}\right\}\right.\right.
\end{aligned}
$$

Fig. 3. How to desequentialize a LCK-derivation of $\Gamma \vdash A$ into a CK-WIS for $\Gamma \vdash A$.

where $h_{i}=h_{\mathrm{p}}-h_{\mathrm{p}_{i}}$ for each $i \in\{0, \ldots, n\}$.

Each view $\mathrm{p}$ induces an equivalence relation $\stackrel{\mathcal{G}_{\mathrm{p}}}{\sim}$ over $V_{\mathcal{G}}$ generated by the relation:

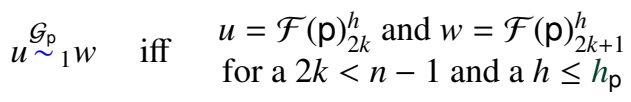

Figure 1 and Equation (1) show four examples of batched views.

Definition 3.4. Let $\mathcal{S}$ be a strategy on a modal arena $\mathcal{G}$. We say that $\mathcal{S}$ is linked if it is well-batched and for every $\mathrm{p} \in \mathcal{S}$ the $\stackrel{\mathcal{G}_{\mathrm{p}}}{\sim}$-classes are of the shape $\left\{v_{1}^{\bullet}, \ldots, v_{n}^{\bullet}, w^{\circ}\right\}$. This induces the edge-relation $u \stackrel{\mathcal{G}_{\mathrm{p}}}{\sim} w$ iff $u^{\bullet} \stackrel{\mathcal{G}_{\mathrm{p}}}{\sim} w^{\circ}$.

We say that $\mathcal{S}$ is CK-batched if it is linked and if for each modal vertex $w^{\circ}$ occurring in the address of a move in $\mathcal{S}$ the following conditions are fulfilled:

i. if $w^{\circ} \in V_{\mathcal{G}}^{\square}$ and $v \stackrel{\mathcal{G}_{\mathrm{p}}}{\rightarrow} w$ for a $\mathrm{p} \in \mathcal{S}$, then $v \in V_{\mathcal{G}}^{\square}$;

ii. if $w^{\circ} \in V_{\mathcal{G}}^{\diamond}$, then there is a unique $u \in V_{\mathcal{G}}^{\diamond}$ in the set $\left\{v \in V_{\mathcal{G}} \mid v \stackrel{\mathcal{G}_{p}}{\rightarrow} w\right.$ for a $\left.\mathrm{p} \in \mathcal{S}\right\}$.

We call a CK-batched WIS a CK-winning innocent strategy (CK-WIS for short).

We can prove that CK-WIS are complete with respect to CK.

Lemma 3.5. If a formula $F$ is provable in LCK, then there is a CK-WIS for $F$.

Proof. For each LCK-derivation $\mathcal{D}$ of $F$ we define the CK-WIS $\{\mathcal{D}\}\}$ by induction over $\mathcal{D}$ according to the rules in Figure 3 In fact, for each rule if its premise $\mathcal{D}^{\prime}$ or both premises $\mathcal{D}_{1}$ and $\mathcal{D}_{2}$ are CK-WISs, then also $\mathcal{D}$ is.

In order to provide sequentialization we prove three preliminary lemmas. The first two lemmas give a way to sequentialize the CK-WISs when a $\wedge$ in the right-hand side 
of the sequent or a $\supset$ in the left-hand side of the sequent occurs. In the sequent calculus LCK these connective require the use of rules splitting the context. In order to avoid to reprove the splitting lemmas from [21], we adopt a simpler approach relying on the presence of $\mathrm{W}$ and $\mathrm{C}$ in the sequent system. The third result proves that the presence of the two rules $\mathrm{K}^{\square}$ and $\mathrm{K}^{\diamond}$ can be easily recognized and sequentialized by only considering the shape of the conclusion sequent and the CK-framing conditions.

Lemma 3.6. Let $\Gamma \vdash A_{1} \wedge A_{2}$ such that $\Gamma$ does not contain $\wedge$-formulas. If $\mathcal{S}$ is a CKWIS for $\Gamma \vdash A_{1} \wedge A_{2}$, then there are CK-WISs $\mathcal{S}_{1}$ and $\mathcal{S}_{2}$ for $\Gamma \vdash A_{1}$ and $\Gamma \vdash A_{2}$.

Proof. For $i \in\{1,2\}$ we let $\mathcal{S}_{i}$ be the set of views in $\mathcal{S}$ starting form a move in $A_{i}$ plus the empty view, that is, $\mathcal{S}_{i}=\left\{\mathrm{p} \in \mathcal{S} \mid \mathrm{p}_{0} \in \vec{R}_{\llbracket A_{i} \rrbracket}\right\} \cup\{\epsilon\}$. By definition of the arena $\llbracket \Gamma \vdash A_{1} \wedge A_{2} \rrbracket$ no move in $A_{i}$ may occur in a view in $\mathcal{S}_{j}$ whenever $i \neq j$. Hence $\mathcal{S}_{1}$ and $\mathcal{S}_{2}$ are CK-WISs for $\Gamma \vdash A_{1}$ and $\Gamma \vdash A_{2}$ respectively.

Lemma 3.7. Let $\mathcal{S}$ be a CK-WIS for $\Gamma^{\prime} \vdash c^{\circ}$, hence $c^{\circ} c^{\bullet} \in \mathcal{S}$. If $\Gamma^{\prime}$ contains no $\wedge$ formulas and $\Gamma^{\prime} \neq \Gamma, c^{\bullet}$, then $\Gamma^{\prime}=\Gamma, A \supset B\left\{c^{\bullet}\right\}$ for a formula $A \supset B\left\{c^{\bullet} \neq c^{\bullet}\right.$ containing the occurrence $c^{\bullet}$ of the atom $c$. Moreover there is a CK-WIS $\mathcal{T}$ for $\Gamma \vdash A$ and $a$ CK-WIS $\mathcal{R}$ for $\Gamma, A \supset B\{c\}, B\{c\} \vdash c$.

Proof. By $\bullet$-determinism and $\bullet$-totality, there is a uniquely determined vertex $c^{\bullet}$ such that $c^{\circ} c^{\bullet} \in \mathcal{S}$. Since $\Gamma^{\prime} \neq \Gamma, c^{\bullet}$ does not contain $\wedge$-formulas, then $\Gamma^{\prime}$ contains a formula $A \supset B\left\{c^{\bullet}\right\}$ and, by definition of WIS, there is a view $c^{\circ} c^{\bullet} v \in \mathcal{S}$ for any $v \in \vec{R}_{\llbracket A \rrbracket}$.

We first show that for a $v \in \vec{R}_{\llbracket A \rrbracket}$ there is a maximal $\sigma \in \mathcal{S}$ such that $v=\sigma_{2 k}$ and $\sigma_{i}$ is not a move in $B\left\{c^{\bullet}\right\}$ for any $i>2 k$. If $k=1$ the property holds. Otherwise, let $i>2 k$ such that $\sigma_{i}$ is the first move in $B\left\{c^{\bullet}\right\}$, hence $\sigma_{i} \in \vec{R}_{\llbracket B\left\{c^{\bullet}\right\} \|}$. By o-completeness, there is a $\sigma^{\prime} \in \mathcal{S}$ such that $\sigma^{\prime}=\sigma_{0} \cdots \sigma_{i} v$. By iterating this reasoning $\mathcal{S}$ should contain a view of infinite length. Hence the property holds.

Now observe that a $\sigma^{v}$ with the previous property exists for a given $v \in \vec{R}_{\llbracket A \rrbracket}$. Thus, by o-completeness, for each $w \in \vec{R}_{\llbracket A \rrbracket}$ there is a $\sigma^{w}$ with the same property and such that $\sigma_{0}^{v} \cdots \sigma_{2 k-1}^{v}=\sigma_{0}^{w} \cdots \sigma_{2 k-1}^{w}$. We define Split ${ }_{\mathcal{S}}^{A}$ to be the set containing such a view $\sigma^{w}$ for each $w \in \vec{R}_{\llbracket A \rrbracket}$. All the $\sigma^{w}$ share the same prefix. We use this Split $_{\mathcal{S}}^{A}$ to define

$$
\begin{aligned}
& \mathcal{T}=\left\{\tau \mid \text { there are } \sigma \text { and } \tau^{\prime} \text { such that } \sigma \tau \tau^{\prime} \in \operatorname{Split}_{\mathcal{S}}^{A}\right\} \\
& \mathcal{R}=\left\{\rho \mid \text { there is no } \sigma \text { such that } \rho \sigma \in \operatorname{Split}_{\mathcal{S}}^{A}\right\}
\end{aligned}
$$

By definition, $\mathcal{T}$ is a CK-WIS for $\Gamma \vdash A$ and $\mathcal{R}$ is a CK-WIS for $\Gamma, A \supset B\{c\}, B\{c\} \vdash c$ strictly smaller than $\mathcal{S}$.

Lemma 3.8. Let $\mathcal{S}$ be a $\mathrm{CK}-\mathrm{WIS}$ s for $\Gamma^{\prime} \vdash A^{\prime}$ such that $\Gamma^{\prime}$ contains no $\wedge$-formulas and at least one move from each formula in $\Gamma^{\prime}$ occurs in a view in $\mathcal{S}$.

- If $A^{\prime}=\square A$, then $\Gamma^{\prime} \vdash A^{\prime}$ is of the form $\square \Gamma \vdash \square A$ and $\mathcal{S}$ is also a CK-WIS for $\Gamma \vdash A$.

- If $A^{\prime}=\diamond A$, then $\Gamma^{\prime} \vdash A^{\prime}$ is of the form $\square \Gamma, \diamond^{\bullet} B \vdash \diamond^{\circ} A$ and $\mathcal{S}=\mathcal{S}^{\prime} \cup\left\{\diamond^{\circ}, \diamond^{\circ} \diamond^{\bullet}\right\}$ where $\mathcal{S}^{\prime}$ is a CK-WIS for $\Gamma \vdash A$. 


\begin{tabular}{|c|c|c|}
\hline Sequent & Shape of $\mathcal{S}$ & Shape of $\mathcal{D}_{\mathcal{S}}$ \\
\hline$\vdash 1$ & $\mathcal{S}=\{\epsilon\}$ & $\bar{r}^{1}$ \\
\hline$a \vdash a$ & $\mathcal{S}=\{\epsilon, a, a a\}$ & $\frac{-}{a+a} \mathrm{AX}$ \\
\hline$\Gamma, B \wedge C \vdash A$ & any & $\frac{\Gamma, B, C \vdash A}{\Gamma, B \wedge C \vdash A} \wedge^{\mathrm{L}}$ \\
\hline$\Gamma \vdash B \supset A$ & any & $\begin{array}{l}\mathcal{D}_{\mathcal{S}} \overline{\|} \\
\frac{\Gamma, B \vdash A}{\Gamma \vdash B \supset A} \supset^{\mathrm{R}} \\
\end{array}$ \\
\hline $\begin{array}{c}\Gamma \vdash A_{1} \wedge A_{2} \\
\Gamma \text { contains no } \wedge \text {-formula }\end{array}$ & $\begin{array}{c}\mathcal{S}=\mathcal{T} \cup \mathcal{R} \\
\mathcal{T}=\left\{\tau \in \mathcal{S} \mid \tau \text { contains no moves in } A_{2}\right\} \\
\mathcal{R}=\left\{\rho \in \mathcal{S} \mid \rho \text { contains no moves in } A_{1}\right\}\end{array}$ & 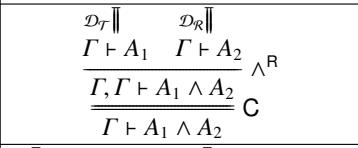 \\
\hline $\begin{array}{c}\Gamma, A \supset B\left\{c^{\bullet}\right\} \vdash c^{\circ} \\
c \text { atomic and } A \supset B\left\{c^{\bullet}\right\} \neq c^{\bullet} \\
B\left\{c^{\bullet}\right\} \text { contains the atom } c^{\bullet} \\
\Gamma \text { contains no } \wedge \text {-formulas }\end{array}$ & $\begin{array}{c}c^{\circ} c^{\bullet} \in \mathcal{S} \\
\mathcal{T}=\left\{\tau \mid \text { there are } \sigma \text { and } \tau^{\prime} \text { such that } \sigma \tau \tau^{\prime} \in \operatorname{Split}_{\mathcal{S}}^{A}\right\} \\
\mathcal{R}=\left\{\rho \mid \text { there is no } \sigma \text { such that } \rho \sigma \in \operatorname{Split}_{\mathcal{S}}^{A}\right\}\end{array}$ & 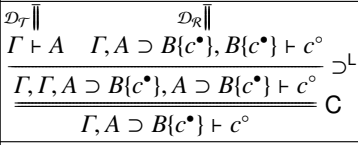 \\
\hline$\Gamma, B \vdash A$ & $\mathcal{S}$ contains no moves in $B$ & $\begin{array}{l}\mathcal{D}_{S} \| \\
\frac{\Gamma \vdash A}{\Gamma, B \vdash A} \mathrm{~W}\end{array}$ \\
\hline$\square \Gamma \vdash \square A$ & $\begin{array}{l}\text { at least one move of each formula } \\
\text { in } \square \Gamma \text { occurs in } \mathcal{S}\end{array}$ & $\begin{array}{l}\mathcal{D}_{S} \overline{\|} \\
\Gamma \Gamma \cdot A \\
\square \Gamma \cdot \square A\end{array}$ \\
\hline$\square \Gamma, \diamond^{\bullet} B \vdash \diamond^{\circ} A$ & $\begin{array}{l}\text { at least one move of each formula } \\
\text { in } \square \Gamma, \diamond^{\bullet} B \text { occurs in } \mathcal{S}\end{array}$ & $\begin{array}{l}\mathcal{D}_{S \backslash \otimes^{\circ}, \otimes^{\circ} \bullet \bullet} \bar{\Pi} \\
\qquad \frac{\Gamma, B \vdash A}{\square \Gamma, \diamond B \vdash \diamond A} \mathrm{~K}^{\diamond}\end{array}$ \\
\hline
\end{tabular}

Fig. 4. Sequentialization procedure

Proof. By the CK-batched condition, if at least one move from each formula in $\Gamma^{\prime}$ occurs in a view in $\mathcal{S}$, then each principal modality of a formula in $\Gamma^{\prime}$ must occur in the first row of a batched view of a $p \in \mathcal{S}$. Moreover, all the principal modalities of the formulas in $\Gamma^{\prime}$ must be in $\stackrel{\mathcal{G}_{\mathrm{p}}}{\longrightarrow}$-relation with the principal modality of $A^{\prime}$ for a $\mathrm{p} \in \mathcal{S}$. Hence $\Gamma^{\prime} \vdash A^{\prime}$ is either of the form $\square \Gamma \vdash \square A$ or $\square \Gamma, \diamond B \vdash \diamond A$. In the first case, we conclude by remarking that if we remove the first row in any batched view $\mathcal{F}(p)$ with $\mathrm{p} \in \mathcal{S}$, then we obtain a batched view of the same $\mathrm{p}$, but in $\Gamma \vdash A$. The second case is similar, but we here have to consider that the strategy also contains the two views $\diamond^{\circ}$ and $\diamond^{\circ} \diamond^{\bullet}$.

We can now prove the following correspondence between CK-proofs and CK-WISs.

Theorem 3.9. Let $F$ be a formula. We have $\stackrel{\text { LCK }}{\models} F$ iff there exists a CK-WIS for $F$.

Proof. It $\mathcal{D}$ is a LCK-derivation of $F$, we can define a CK-WIS $\mathcal{S}_{\mathcal{D}}$ for $F$ as in Lemma 3.5 To prove the converse, we define a LCK-derivation $\mathcal{D}_{S}^{\Gamma+A}$ for the sequent $\Gamma \vdash A$ by induction on the lexicographic order on the triple $\langle|\mathcal{S}|,\|A\|,\|\Gamma\|\rangle$. We remark that if in no view in a CK-WIS $\mathcal{S}$ for $\Gamma, B \vdash A$ contains moves in $B$, then $\mathcal{S}$ is a CK-WIS also for $\Gamma \vdash A$. Observe that in case of $\diamond$-formulas occurring in $\Gamma$, we may have that only one of these $\diamond$ s occurring in a view. In this case, we expect to observe in the final derivation a $\mathrm{K}^{\diamond}$-rule preceded (bottom-up) by a $\mathrm{W}$-rule. 
Moreover, since $\llbracket \Gamma, B \wedge C \vdash A \rrbracket=\llbracket \Gamma, B, C \vdash A \rrbracket$, then each CK-WIS for the first sequent is a CK-WIS for the second one, but the size of the lhs sequent decreases. A similar reasoning applies to the sequents $\Gamma \vdash B \supset C$ and $\Gamma, B \vdash C$. We conclude by Lemmas 3.6, 3.7 and 3.8.

In Figure 4 we give a table resuming the sequentialization step to apply according to the shape of the sequent and the shape of the CK-WIS. The conditions on the sequent (first column) can be checked in the given order, triggering the corresponding sequentialization step.

\section{Compositionality of Winning Strategies}

In order to simplify the presentation of our compositionality result, we propose a slightly different approach to the proof of winning strategies compositionality with respect to the one normally used in the literature, e.g. [1117], where proofs are given by reasoning on specific sequences ${ }^{6}$ over the arena $\llbracket A \supset(B \supset C) \rrbracket \stackrel{f}{\sim} \llbracket A, B \vdash C \rrbracket$, such that these views can be projected on views over the arenas of $A \vdash B$ and $B \vdash C$. Instead, we here reason directly over (non o-shortsighted) views over the arena $\llbracket A, B \supset B \vdash C \rrbracket$. This allows us to preserve the parities of vertices when performing the projections.

To obtain an intuition behind the idea, consider the additional rule hide removing a formula of the shape $B \supset B$ occurring in the left-hand side of a sequent in order to simulate the cut as shown below.

$$
\frac{\Gamma \vdash B \quad \Delta, B \vdash C}{\Gamma, \Delta \vdash C} \text { cut } \leadsto \frac{\Gamma \vdash B \quad B, \Delta \vdash C}{\frac{\Gamma, \Delta, B \supset B \vdash C}{\Gamma, \Delta \vdash C}} \supset^{\mathrm{L}} \text { hide }
$$

This approach complies with the slogan "interaction + hide" often mentioned in the literature, e.g., [117]. Here the interaction is represented by the $\supset^{\mathrm{L}}$-rule, while the hiding is performed by erasing the formula $A \supset A$ using the hide-rule.

In terms of views, our interaction is defined by composing views from the two corresponding strategies by "gluing" them using a copycat strategy on the cut-formula while the hiding consist of ignoring the moves in the hidden formulas.

Notation 4.1. If $\Delta$ is a list (of occurrences) of formulas in $\Gamma \vdash A$ and $\mathrm{p}$ is a sequence of moves in $\llbracket \Gamma \vdash A \rrbracket$, we denote by $\mathrm{p}_{\Delta}$ the projection of $\mathrm{p}$ on $\Delta$, that is, the sequence obtained by erasing from $\mathrm{p}$ any move not in $\Delta$. By means of example, if $A=a \supset e$, $B=b \wedge d$ and $C=c$, then baadcebda $\left.\right|_{A, C}=$ aacea .

Whenever we consider two distinct occurrences $B_{1}$ and $B_{2}$ of the same formula $B$, we assume ${ }^{\perp}$ to be the bijection between the vertices in $V_{\llbracket B_{1} \rrbracket}$ and in $V_{\llbracket B_{2} \rrbracket}$ corresponding to the same occurrence of the atom or modality in $B$. Note that $b$ is a $\circ$-move (resp. $\bullet-$ move) in $B_{1}$ iff $b^{\perp}$ is an •-move (resp. o-move) in $B_{2}$.

Definition 4.2. Let $\mathcal{T}$ and $\mathcal{R}$ be WISs respectively for $A \vdash B_{1}$ and $B_{2} \vdash C$ such that $B_{1}$ and $B_{2}$ are occurrences of the same formula $B$, and let $\tau \in \mathcal{T}$ and $\left.\rho \in \mathcal{R}\right]^{7}$

\footnotetext{
${ }^{6}$ Note that these sequences are not views.

${ }^{7}$ Note that a CK-WIS for $\Gamma, A \vdash B$ is also a CK-WIS for $A \vdash(\bigwedge \Gamma) \supset B$. This allows us to consider only CK-WISs for sequents of the shape $A \vdash B$.
} 
We define the interaction of $\tau$ ad $\rho$ over $B$ as the sequence of moves $\sigma=\tau \stackrel{B}{\circ} \rho$ over $\llbracket A, B_{1} \supset B_{2} \vdash C \rrbracket$ following $\rho$ (resp. $\tau$ ) until a $\bullet$-move $b$ in $B_{2}$ (resp. $B_{1}$ ) is reached; then it switches to the corresponding o-move $b^{\perp}$ in $\tau$ (resp. $\rho$ ), if it exists. That is,

$$
\sigma_{0}=\rho_{0} \text { and } \sigma_{i+1}= \begin{cases}\tau_{k+1} & \text { where } \sigma_{i}=\tau_{k} \text { is a move in } A \text { or a o-move in } B_{1} \\ \rho_{k+1} & \text { where } \sigma_{i}=\rho_{k} \text { is a move in } C \text { or a o-move in } B_{2} \\ b^{\perp} & \text { where } \sigma_{i}=b \text { is a } \bullet \text {-move in } B_{1} \text { and } b^{\perp} \text { occurs in } \rho \\ b^{\perp} & \text { where } \sigma_{i}=b \text { is a } \bullet \text {-move in } B_{2} \text { and } b^{\perp} \text { occurs in } \tau \\ \text { not defined } & \text { otherwise }\end{cases}
$$

We define the composition $\tau^{B} * \rho$ of $\tau$ and $\rho$ over $B$ as the projection of $\tau \stackrel{B}{\circ} \rho$ over $A$ and $C$, that is, $\tau *{ }^{B} \rho=\left.(\tau \stackrel{B}{\bullet} \rho)\right|_{A, C}$. We define the composition of $\mathcal{T}$ and $\mathcal{R}$ over $B$ as the following set of sequences over $\llbracket A \vdash C \rrbracket$

$$
\mathcal{T}^{B} \mathcal{R} \mathcal{R}=\left\{\tau^{B} * \rho \mid \tau \in \mathcal{T}, \rho \in \mathcal{R}\right\}
$$

Intuitively, when defining the interaction $\mathrm{o}$, the player $\circ$ changes way to play: whenever the player $\bullet$ plays a move $b$ in $B_{1}$ (or $B_{2}$ ), its successive o-move is the corresponding $b^{\perp}$ in $B_{2}$ (resp. $B_{1}$ ) instead of playing according to o-shortsightedness. By definition $\left.(\tau \stackrel{B}{\ominus} \rho)\right|_{A, B_{1}}=\tau$ and $\left.(\tau \stackrel{B}{*} \rho)\right|_{B_{2}, C}=\rho$, hence $(\tau \stackrel{B}{\bullet} \rho)$ is always finite. The rest of this section is devoted to prove that if $\mathcal{T}$ and $\mathcal{R}$ are CK-WISs, then also is $\mathcal{T}^{B} * \mathcal{R}$.

Example 4.3. Consider the sequents $A \vdash B_{1}=(c \supset a) \supset b \vdash(d \wedge(c \supset a)) \supset b$ and $B_{2} \vdash C=(d \wedge(c \supset a)) \supset b \vdash(d \wedge((e \supset e) \supset a)) \supset b$ and the view $\tau=$ bbaacc on $\llbracket A \vdash B_{1} \rrbracket$ and the views $\rho_{1}=$ bbaaee and $\rho_{2}=$ bbdd on $\llbracket B_{2} \vdash C \rrbracket$. Note that these views are the unique maximal views in the unique WISs for these sequents. Then we can picture the construction of $\tau *{ }^{B} \rho_{1}$ as follows, where on the left-hand side we highlight the two occurrences of $\llbracket B \rrbracket$ on which the views interact, and the black arrows identify the sequences of moves on the arenas.

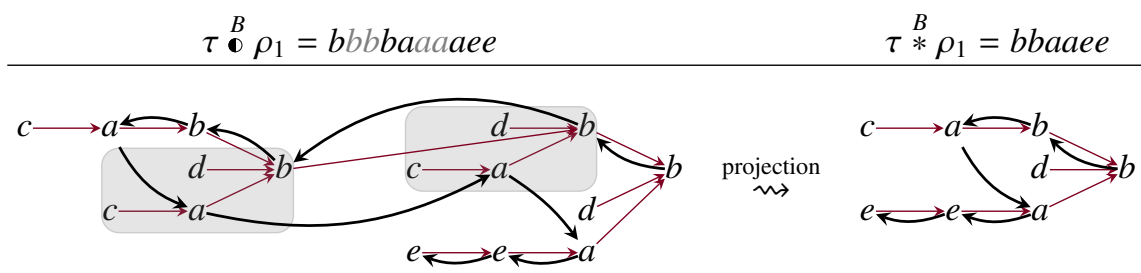

Similarly $\tau \stackrel{B}{\circ} \rho_{2}=b b b b a a$ and $\tau \stackrel{B}{*} \rho_{2}=b b a$. Note that in this case the definition of $\tau{ }^{B} \rho_{2}$ stops because the successive should be $a^{\perp}$ but it does not occur in $\rho$.

Remark 4.4. If $A, B_{1}, B_{2}$ and $C$ are formulas with $B_{1}$ and $B_{2}$ occurrences of the same formula $B$, then atoms and modalities in these formulas have the same parity in $\llbracket A, B_{1} \supset B_{2} \vdash C \rrbracket$ and in $\llbracket A \vdash B_{1} \rrbracket$ and $\llbracket B_{2} \vdash C \rrbracket$.

Our definitions allow us to show that the composition of well-batched WISs is a well-batched WIS. 
Lemma 4.5. Let $\mathcal{T}$ and $\mathcal{R}$ be well-batched WIS for respectively $A \vdash B_{1}$ and $B_{2} \vdash C$ such that $B=B_{1}=B_{2}$. Then $\mathcal{S}=\mathcal{T}^{*} \mathcal{R}$ is a well-batched WIS for $A \vdash C$.

Proof. We first prove that that for each $\tau \in \mathcal{T}$ and $\rho \in \mathcal{R}$ we have that $\tau{ }^{*} \rho$ is a well-batched view over $\llbracket A \vdash C \rrbracket$ since it verifies all conditions in Definition 3.1 For any $\sigma=\tau * \stackrel{B}{*} \rho$ we have that

1. $\sigma$ is a play: since $\sigma_{0} \in \vec{R}_{\llbracket B \vdash C \rrbracket}$ and $\vec{R}_{\llbracket B \vdash C \rrbracket}=\vec{R}_{\llbracket C \rrbracket}=\vec{R}_{\llbracket A \vdash C \rrbracket}$.

2. $\sigma$ is justified: if a move in $\llbracket A \rrbracket$ is justified in $\tau$ by a move in $\llbracket A \rrbracket$ or if a move in $\llbracket C \rrbracket$ is justified in $\rho$ by a move in $\llbracket C \rrbracket$, then we can conclude. By definition of $\llbracket B \vdash C \rrbracket$ no move in $\llbracket C \rrbracket$ can be justified in $\rho$ by a move in $B$. We conclude by remarking that if a move in $\llbracket A \rrbracket$ is justified in $\tau$ by a move in $\llbracket B \rrbracket$, then this move must be a root of $\llbracket A \rrbracket$, and then $v \stackrel{\llbracket A \vdash C \rrbracket}{\rightarrow} \sigma_{0}$ since $u \stackrel{\llbracket A \vdash C \rrbracket}{\rightarrow} w$ for all $u \in \vec{R}_{\llbracket A \rrbracket}$ and $w \in \vec{R}_{\llbracket C \rrbracket}$.

3. $\sigma$ is o-shortsighted: by definition of $\sigma$ we must have that both $\sigma_{2 k+1}$ and $\sigma_{2 k+2}$ are either in $\llbracket A \rrbracket$ or in $\llbracket C \rrbracket$. We conclude by hypothesis on $\rho$ and $\tau$.

4. $\sigma$ is $\bullet$-uniform: by induction using the $\bullet$-uniformity of $\tau$ and $\rho$ and the fact that $\ell(v)=\ell\left(v^{\perp}\right)$. In fact, let $\tilde{\sigma}=\tau \stackrel{B}{\bullet} \rho$. If $\tilde{\sigma}_{i}$ is a move in $A$ (in $C$ ), $\tilde{\sigma}_{i+1} \cdots \tilde{\sigma}_{i+k-1}$ are moves over $B_{1}$ and $B_{2}$, and $\tilde{\sigma}_{i+k}$ is a move in $C$ (respectively in $A$ ), then we can prove by induction that $\ell\left(\tilde{\sigma}_{i}\right)=\ell\left(\tilde{\sigma}_{i+j}\right)$ for all $j \in\{1, \ldots, k\}$.

5. $\sigma$ is modal: follows by the fact that no move in $\tau$ or $\rho$ is a $\square$-vertex.

6. $\sigma$ is well-batched: it suffices to remark that if $\operatorname{add}_{v}=m_{1} \cdots m_{k}$, then $\operatorname{add}_{v^{\perp}}=$ $m_{1}^{\perp} \cdots m_{k}^{\perp}$. We can conclude similarly to the proof of $\bullet$-uniformity since in $\tau \stackrel{B}{\bullet} \rho$ in all moves in a subsequence in $B_{1}$ and $B_{2}$ have constant height.

To conclude we show that that $\mathcal{S}$ is

a. predecessor-closed: it follows by the fact that $\mathcal{T}^{B} \mathcal{R}=\{\tau \stackrel{B}{\circ} \rho \mid \tau \in \mathcal{T}, \rho \in \mathcal{R}\}$ is predecessor closed.

b. o-complete: if $\sigma v^{\bullet} \in \mathcal{S}$ then $v^{\bullet}$ appears in a view $\tau \in \mathcal{T}$ or in a view $\rho \in \mathcal{R}$ as an --move. We conclude by the definition of the composition $*$ and by the fact that $\mathcal{S}$ and $\mathcal{R}$ are WIS.

c. •-deterministic and •-total: by induction on the length of $\tau \stackrel{B}{\bullet} \rho$ we can prove that each $v^{\circ} \in \tau \stackrel{B}{\bullet} \rho$ is followed by a unique $\bullet$-move since $\mathcal{T}$ and $\mathcal{R}$ are $\bullet$-deterministic and each $v^{\perp} \in \llbracket B_{1} \rrbracket$ and $w^{\perp} \in \llbracket B_{2} \rrbracket$ is uniquely determined respectively by a $v \in$ $\llbracket B_{2} \rrbracket$ and a $w \in \llbracket B_{1} \rrbracket$. If $\bullet$-totality does not hold, then there should be a maximal view in $\mathcal{S}$ of odd length. That is, there should be $\tau \in \mathcal{T}$ and $\rho \in \mathcal{R}$ such that $\tau$ B $\rho=\tilde{\sigma}=\tilde{\sigma}^{\prime} v^{\circ} s$ for a $v^{\circ}$ move in $A$ or $C$, and a sequence $s$ of moves in $B_{1}$ or in $B_{2}$. By $\bullet$-totality of $\mathcal{T}$ and $\mathcal{R}$ we can assume that $\tilde{\sigma}$ terminates with an $\bullet$-move $\tilde{\sigma}_{n}$. Moreover, $\tilde{\sigma}_{n}$ would be justified by a o-move $\tilde{\sigma}_{h}$ in $B_{1}$ or $B_{2}$ such that $\tilde{\sigma}_{h-1}=\tilde{\sigma}_{h}^{\perp}$. Thus we could find $\tau^{\prime} \in \mathcal{T}$ and $\rho^{\prime} \in \mathcal{R}$ such that $\tau^{\prime} \stackrel{B}{\bullet} \rho^{\prime}=\tilde{\sigma}_{1} \ldots \tilde{\sigma}_{n} \tilde{\sigma}_{n}^{\perp}$, contradicting the maximality of $\tilde{\sigma}$.

We can now prove that the composition of CK-WISs is a CK-WIS.

Theorem 4.6. Let $\mathcal{T}$ and $\mathcal{R}$ be CK-WIS for respectively $A \vdash B_{1}$ and $B_{2} \vdash C$ such that $B_{1}$ and $B_{2}$ are occurrences of the same formula $B$. Then $\mathcal{S}=\mathcal{T}^{B} \mathcal{R}$ is a CK-WIS. 
Proof. After Lemma 4.5 it suffices to prove that $\mathcal{S}$ is CK-batched 8 . To improve read-

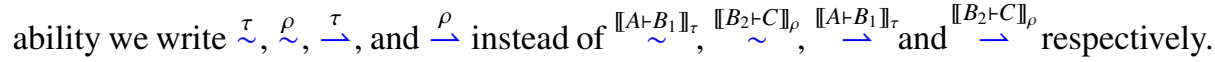

For this purpose we define for each $\sigma=\tau * \rho$ the relation $\stackrel{\tau \oplus \rho}{\longrightarrow}$ on the vertices in $\llbracket A, B_{1} \supset B_{2} \vdash C \rrbracket$ as the transitive closure of the following relation

$$
v \stackrel{\tau \oplus \rho}{\rightarrow}{ }_{1} w \Longleftrightarrow v^{\bullet} \stackrel{\tau}{\rightarrow} w^{\circ} \text { or } v^{\bullet} \stackrel{\rho}{\rightarrow} w^{\circ} \text { or } v=w \text { or } v^{\circ}=\left(w^{\bullet}\right)^{\perp}
$$

where we write $w^{\llcorner}\left\llcorner v\right.$ if $v^{\circ}=\left(w^{\bullet}\right)^{\perp}$.

We use $\stackrel{\tau \bullet \rho}{\longrightarrow}$ to prove the properties of $\stackrel{\sigma}{\sim}$. In particular, if $v v_{\sim}^{\tau} w$ in $V_{\llbracket A \rrbracket}$ or $v \stackrel{\rho}{\sim}{ }_{1} w$ in $V_{\llbracket C \rrbracket}$, then $v \stackrel{\sigma}{\sim} 1 w$. Observe that if $\mathcal{T}$ and $\mathcal{R}$ are linked, then $\stackrel{\tau}{\rightarrow}$ and $\stackrel{\rho}{\rightarrow}$ can be considered as functions associating a vertex $v^{\bullet}$ a unique vertex $w^{\circ}$. Then also $\stackrel{\tau}{\longrightarrow} \rho$ can be considered as a function since $\stackrel{\perp}{\llcorner}$ is a bijection.

If a $\stackrel{\sigma}{\sim}$-class contains only vertices either in $A$ or in $C$, then we can conclude by hypothesis on $\mathcal{T}$ and $\mathcal{R}$. Otherwise, we only prove the case when $v \stackrel{\tau \oplus \rho}{\longrightarrow} w$ with $v \in V_{\llbracket A \rrbracket}$ and $w \in V_{\llbracket C \rrbracket}$ since the case with $v \in V_{\llbracket C \rrbracket}$ and $w \in V_{\llbracket A \rrbracket}$ is proven symmetrically. By definition of $v \stackrel{\tau \bullet}{\rightarrow} w$ we have $\tau \bullet \rho=\gamma_{0} \cdots \gamma_{i} \beta_{0} \cdots \beta_{k} \alpha_{0} \cdots \alpha_{j}$ with $w=\operatorname{add}_{\gamma_{i}}^{h}$ and $v=\operatorname{add}_{\alpha_{0}}^{h}$, and $b_{1} \ldots, b_{n} \in V_{\llbracket B_{2} \rrbracket}$ such that in the batched view of $\tau \rho$ we have the columns below, where at the bottom of each column we annotated the player playing the move and the formula in which the move and the vertices in its address belong.

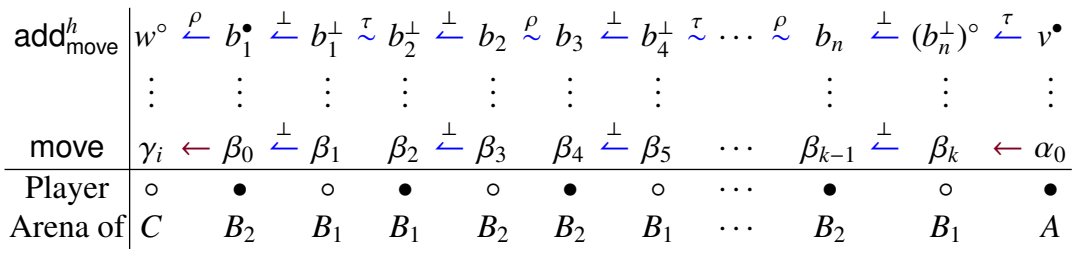

The proof that Conditions i and ii from Definition 3.4 hold follows by a similar reasoning on $\stackrel{\tau \otimes}{\rightarrow}$ using the fact that $\mathcal{T}$ and $\mathcal{R}$ are CK-batched. More precisely, if $\ell(v)=\diamond$ or $\ell(w)=\square$, then we have $\ell(v)=\ell\left(b_{i}\right)=\ell\left(b_{i}^{\perp}\right)=\ell(w)$ for each $i \in\{1, \ldots, n\}$.

Lemma 4.7. Let $A, B, C$ and $D$ formulas. If $\mathcal{S}$ is $a$ CK-WIS for $A \vdash B$ and $\mathcal{T}$ is $a$ CK-WIS for $B \vdash C$ and $\mathcal{R}$ is a CK-WIS for $C \vdash D$, then $\left(\mathcal{S}{ }_{*}^{B} \mathcal{T}\right){ }^{C} \mathcal{R}=\mathcal{S}^{B}\left(\mathcal{T}^{C} \mathcal{R}\right)$.

Proof. The operation $\bullet$ is associative by Definition 4.2. Moreover, for any $\Delta$ and $\Sigma$ sequences of formulas, the projections on $\Delta$ and $\Sigma$ permute, that is, $\left.\left(\left.s\right|_{\Delta}\right)\right|_{\Sigma}=\left.s\right|_{4, \Sigma}=$ $\left.\left(\left.s\right|_{\Sigma}\right)\right|_{\Delta}$. We conclude by observing that for any $\sigma \in \mathcal{S}, \tau \in \mathcal{T}$ and $\rho \in \mathcal{R}$ we have

$$
\begin{aligned}
& \sigma^{B} *\left(\tau^{C} * \rho\right)=\left.\left(\sigma^{A}\left(\tau^{B} * \rho\right)\right)\right|_{A, D}=\left.\left(\sigma_{\bullet}^{B}\left(\left.\left(\tau_{\bullet}^{C} \rho\right)\right|_{B, D}\right)\right)\right|_{A, D}= \\
& =\left.(\sigma \stackrel{B}{\bullet}(\tau \stackrel{C}{\bullet} \rho))\right|_{A, D}=\left.\left(\left(\sigma \sigma^{B} \tau\right) \stackrel{C}{\bullet} \rho\right)\right|_{A, D}= \\
& =\left.\left(\left(\left.(\sigma \stackrel{B}{\bullet} \tau)\right|_{A, C}\right) \stackrel{C}{\bullet} \rho\right)\right|_{A, D}=\left((\sigma * \tau){\stackrel{C}{\complement} \rho)\left.\right|_{A, D}=(\sigma * \tau)}^{B}{ }^{C} \rho\right.
\end{aligned}
$$

\footnotetext{
${ }^{8}$ This proof is similar to the one in [20].
} 


\section{Game Semantics for Constructive Modal Logic}

In the previous sections, we provide various results on the correspondence between proofs in CK and CK-WISs, as well as the compositionality properties of the latter. This allows us to formally state our full completeness result.

Theorem 5.1. The CK-WISs form a full-complete denotational semantics for CK.

Proof. Let us consider the well-defined map

$$
\{[\cdot\}:\{\text { LCK-derivations of } F\} \rightarrow \quad\{\text { CK-WISs for } F\}
$$

from the proof of Lemma 3.5 After Theorem 3.9 we know that there is a map

$$
\mathcal{D}_{(\cdot)}^{F}:\{\text { CK-WISs for } F\} \quad \rightarrow \quad\{\text { LCK-derivations of } F\}
$$

which guarantees that each CK-WIS is the image of a LCK-derivation. In particular we have that $\left\{\left\{\mathcal{D}_{S}^{F}\right\}=\mathcal{S}\right.$, i.e., the map $\left\{[\cdot\}\right.$ is the left-adjoint of $\mathcal{D}_{(\cdot)}^{F}$. In Theorem 4.6 we prove that CK-WISs compose. Moreover, by Lemma 4.7 composition is associative with neutral element the trivial strategy.

\subsection{Game semantics for CD}

The results presented in this paper can be straightforwardly extended to the constructive modal logic $C D$, which is obtained by extending $C K$ with the modal axiom $d$ shown below left:

$$
\mathrm{d}: \square A \supset \diamond A \quad \frac{\Gamma \vdash A}{\square \Gamma \vdash \diamond A} \mathrm{D}
$$

A sound and complete (cut-free) sequent system for this logic can be obtained by adding the sequent rule above on the right to the sequent system for CK.

In order to define WIS capturing proofs in CD we need some additional definitions.

Definition 5.2. Let $\mathcal{S}$ be a WIS over an arena $\mathcal{G}$. We say that $\mathcal{S}$ is CD-batched if it is atomic, that is, the views in $\mathcal{S}$ contains only atomic vertices, linked, and if for each modal vertex $w^{\circ}$ occurring in the address of a move in $\mathcal{S}$ the following conditions are fulfilled:

i. if $w^{\circ} \in V_{\mathcal{G}}^{\square}$ and $v \stackrel{\mathcal{G}_{\mathrm{p}}}{\rightarrow} w$ for a $\mathrm{p} \in \mathcal{S}$, then $v \in V_{\mathcal{G}}^{\square}$;

ii. if $w^{\circ} \in V_{\mathcal{G}}^{\diamond}$, then there is at most a $u \in V_{\mathcal{G}}^{\diamond}$ in the set $\left\{v \in V_{\mathcal{G}} \mid v \stackrel{\mathcal{G}_{p}}{\rightarrow} w\right.$ for a $\left.p \in \mathcal{S}\right\}$.

Note that the first condition is the same first condition from Definition 3.4 The reason why we do not need the information about the diamonds in the strategies for CD depends on a property of the logic (see [2, Theorem 2]). The idea is that an instance of weakening can permute below $\mathrm{K}^{\diamond}$-rules, transforming it into a an instance of the $\mathrm{D}$-rule, as shown below (while in CK the information about the left-hand side diamond must be kept in some way): 


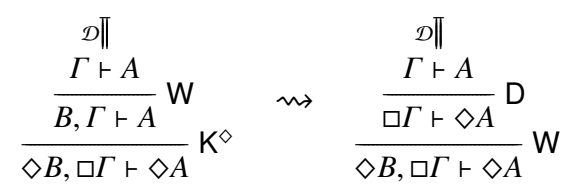

We then define a CD-WIS as a CD-batched WIS. This allows to extend Theorems 3.9 and 4.6 with no effort, that is

Theorem 5.3. The CD-WISs form a full-complete denotational semantics for $\mathrm{CK}$.

\section{Conclusion and Future Work}

In this paper we have defined a game semantics for the constructive modal logic CK and have shown how it can be extended for the logic CD. We have proved full completeness and compositionality of our winning strategies, and thus have shown that our model provides a full complete denotational semantics for CK and CD.

We are currently investigating the possibility of extending our semantics to the logics CT and CS4, that are obtained by adding the modal axioms

$$
\mathrm{T}:(A \supset \diamond A) \wedge(\square A \supset A) \quad \text { and } \quad 4:(\diamond \diamond A \supset \diamond A) \wedge(\square A \supset \square \square A)
$$

However, the problem that arises is that for these logics also the $\square$ should be allowed as move in order to keep track of the rules for $T$ and 4 . However, the •-determinism of winning strategies depends on the fact that atoms and diamonds are paired by the rules which introduce them. This means that when boxes are allowed as moves, determinism cannot hold. We have to leave this issue for future work.

It is worth noticing that our result is strongly related to the game semantics for light linear logic as defined in [21]. In future work we will investigate the relation between our approach and this latter in order to provide a game semantics for elementary and light linear logic.

Finally, we conjecture the existence of a one-to-one correspondence between our CK-WISs and the $\lambda$-calculi for constructive modal logics [3/4/12]. This investigation will also be object of future research.

Acknowledgements We would like to thank Christian Fermüller, Robert Freiman, Yoni Zohar, and anonymous referees for the useful feedback which helped us to improve the final version of the manuscript.

\section{References}

1. Abramsky, S., Malacaria, P., Jagadeesan, R.: Full abstraction for pcf. In: International Symposium on Theoretical Aspects of Computer Software. pp. 1-15. Springer (1994)

2. Acclavio, M., Catta, D., Straßburger, L.: Towards a Denotational Semantics for Proofs in Constructive Modal Logic (Apr 2021), https://hal.archives-ouvertes.fr/ hal-03201439, preprint 
3. Bellin, G., De Paiva, V., Ritter, E.: Extended Curry-Howard correspondence for a basic constructive modal logic. In: In Proceedings of Methods for Modalities (05 2001)

4. Bierman, G.M., de Paiva, V.C.: On an intuitionistic modal logic. Studia Logica 65(3), 383416 (2000)

5. Brünnler, K.: Deep sequent systems for modal logic. Archive for Mathematical Logic 48(6), 551-577 (2009)

6. Davies, R., Pfenning, F.: A modal analysis of staged computation. Journal of the ACM (JACM) 48(3), 555-604 (2001)

7. Fairtlough, M., Mendler, M.: Propositional lax logic. Information and Computation 137(1), 1-33 (1997)

8. Fitch, F.B.: Intuitionistic modal logic with quantifiers. Portugaliae mathematica 7(2), 113 118 (1948)

9. Heilala, S., Pientka, B.: Bidirectional decision procedures for the intuitionistic propositional modal logic IS4. In: International Conference on Automated Deduction. pp. 116-131. Springer (2007)

10. Horne, R., Ahn, K.Y., Lin, S.w., Tiu, A.: Quasi-open bisimilarity with mismatch is intuitionistic. In: Proceedings of the 33rd Annual ACM/IEEE Symposium on Logic in Computer Science. p. 26-35. LICS '18, Association for Computing Machinery, New York, NY, USA (2018). https://doi.org/10.1145/3209108.3209125 https://doi .org/10.1145/ 3209108.3209125

11. Hyland, J., Ong, C.H.: On full abstraction for PCF: I, II, and III. Information and Computation 163(2), 285 - 408 (2000). https://doi.org/https://doi.org/10.1006/inco.2000.2917. http://www.sciencedirect.com/science/article/pii/S0890540100929171

12. Kakutani, Y.: Call-by-name and call-by-value in normal modal logic. In: Shao, Z. (ed.) Programming Languages and Systems. pp. 399-414. Springer Berlin Heidelberg, Berlin, Heidelberg (2007)

13. Kojima, K.: Semantical study of intuitionistic modal logics. Ph.D. thesis, Kyoto University (2012), ph.D. thesis

14. Kuznets, R., Marin, S., Straßburger, L.: Justification logic for constructive modal logic *. IMLA 2017 - 7th Workshop on Intuitionistic Modal Logic and Applications (Jul 2017), https://hal.inria.fr/hal-01614707

15. Lamarche, F.: Proof Nets for Intuitionistic Linear Logic: Essential Nets (2008), https:// hal.inria.fr/inria-00347336

16. Marin, S., Straßburger, L.: Label-free Modular Systems for Classical and Intuitionistic Modal Logics. In: Advances in Modal Logic 10 (2014)

17. McCusker, G.: Games and full abstraction for FPC. Information and Computation 160(1), 1 - 61 (2000). https://doi.org/https://doi.org/10.1006/inco.1999.2845. http://www. sciencedirect.com/science/article/pii/S0890540199928456

18. Mendler, M., Scheele, S.: Cut-free gentzen calculus for multimodal CK. Information and Computation 209(12), 1465-1490 (2011)

19. Meyer, J.J., Veltmanw, F.: Intelligent agents and common sense reasoning. In: Blackburn, P., Van Benthem, J., Wolter, F. (eds.) Handbook of Modal Logic, Studies in Logic and Practical Reasoning, vol. 3, pp. 991 - 1029. Elsevier (2007). https://doi.org/https://doi.org/10.1016/S1570-2464(07)80021-8 ht //wWw.sciencedirect.com/science/article/pii/S1570246407800218

20. Murawski, A.S.: On semantic and type-theoretic aspects of polynomial-time computability (2001)

21. Murawski, A.S., Luke Ong, C.H.: Evolving games and essential nets for affine polymorphism. In: Abramsky, S. (ed.) Typed Lambda Calculi and Applications. pp. 360-375. Springer Berlin Heidelberg, Berlin, Heidelberg (2001) 
22. Plotkin, G., Stirling, C.: A framework for intuitionistic modal logics. In: Proceedings of the 1st Conference on Theoretical Aspects of Reasoning about Knowledge (TARK). pp. 399406 (1986)

23. Prawitz, D.: Natural deduction: A proof-theoretical study. Courier Dover Publications (2006)

24. Simpson, A.K.: The proof theory and semantics of intuitionistic modal logic. Ph.D. thesis, University of Edinburgh. College of Science and Engineering (1994)

25. Straßburger, L.: Cut elimination in nested sequents for intuitionistic modal logics. In: Pfenning, F. (ed.) FoSSaCS'13. LNCS, vol. 7794, pp. 209-224. Springer (2013)

26. Straßburger, L., Heijltjes, W., Hughes, D.J.D.: Intuitionistic proofs without syntax. In: LICS 2019 - 34th Annual ACM/IEEE Symposium on Logic in Computer Science. pp. 1-13. IEEE, Vancouver, Canada (Jun 2019). https://doi.org/10.1109/LICS.2019.8785827. https://hal.inria.fr/hal-02386878

27. Troelstra, A.S., Schwichtenberg, H.: Basic proof theory, vol. 43. Cambridge University Press (2000)

28. Vakarelov, D.: Modal logics for knowledge representation systems. Theor. Comput. Sci. 90, 433-456 (01 1991) 